\title{
Artérias mesentéricas cranial e caudal em aves (Gallus gallus) da linhagem Cobb 500
}

Danila Barreiro CAMPOS 1

Frederico Ozanam Carneiro e SILVA ${ }^{2}$

Renato Souto SEVERINO²

Sérgio Salazar

DRUMMOND 2

Eduardo Maurício Mendes

de LIMA $^{1}$

Marcelo Ismar Silva

SANTANA ${ }^{3}$

Pedro Primo BOMBONATO 1

Correspondência para:

FREDERICOOZANAMCARNEIROESILVA

Departamento de Morfologia

Faculdade de Medicina Veterinária

UniversidadeFederal de Uberlândia

Av. Pará, 1720 - Campus Umuarama

38400-902 - Uberlândia - MG

frederico@famev.ufu.br

Recebido para publicação: 12/05/2004

Aprovado para publicação: 21/09/2005

\author{
1 - Departamento de Cirurgia da Faculdade de Medicina Veterinária e \\ Zooteccnia da Universidade de São Paulo, São Paulo - SP \\ 2 - Departamento de Morfologia da Faculdade de Medicina Veterinária da \\ Universidade Federal de Uberlândia, Uberlândia - MG \\ 3 - Universidade de Franca, Franca - SP
}

\section{Resumo}

Estudaram-se as origens, ramificações e distribuições das artérias mesentéricas cranial e caudal em 30 aves (Gallus gallus) da linhagem Cobb 500, machos, com idade entre 5 e 6 semanas. Os espécimes tiveram seus vasos arteriais preenchidos com solução aquosa corada de Neoprene Látex " 450 ” a 50\% e a seguir foram fixados em solução aquosa de formaldeído a 10\%. A artéria mesentérica cranial emergiu da face lateral direita da aorta descendente caudalmente à artéria celíaca e através das artérias ileocecal, jejunais e ileais distribuiu-se pelo jejuno, íleo, cecos e reto, terminando próximo ao divertículo vitelino. A artéria mesentérica caudal originou-se da face ventral da aorta descendente e após curto trajeto bifurcou-se em ramos cranial e caudal, distribuindose pelo reto, cloaca e bolsa cloacal. Evidenciaram-se anastomoses entre ramos da artéria mesentérica cranial e entre ramos desta e ramos das artérias mesentérica caudal e celíaca.

\section{Introdução}

A atual avicultura industrial teve um grande impulso em seu desenvolvimento logo após a segunda guerra mundial ${ }^{1}$, sendo que as constantes exigências de aumentos da produção aliadas a necessidade de diminuição dos custos, impulsionam investimentos tecnológicos nas áreas de genética, manejo, sanidade, nutrição e ambiência ${ }^{2}$.

A modernização da avicultura através do melhoramento genético teve como característica inicial o desenvolvimento de híbridos e linhagens mais refinadas ${ }^{3}$. Foi pela necessidade de um produto com conformação superior e alta conversão alimentar, que através do cruzamento de fêmeas White Rock e machos Vantress iniciou-se a produção das aves da linhagem Cobb 500, cujos frangos de corte, após um rigoroso processo de seleção, adquiriram características como rápido crescimento, eficiência alimentar, baixa mortalidade e alto rendimento de carcaça ${ }^{4}$.
No que diz respeito à morfologia das aves, ainda que toda a classe apresente as menores variações quanto a forma, quando comparada a muitas ordens de peixes, anfibios, répteis e até mesmo de mamíferos ${ }^{5}$, fatores como o cruzamento e a seleção podem assumir fundamental importância no aumento das variações anatômicas ${ }^{6}$.

Desta forma, a presente investigação científica teve como objetivo fornecer informações pertinentes às origens, ramificações e distribuições das artérias mesentéricas cranial e caudal em aves da linhagem Cobb 500, oferecendo ainda subsídios que possam contribuir para o conhecimento destes aspectos morfológicos em Gallus gallus e nas demais espécies de aves.

\section{Materiais e Métodos}

Utilizaram-se 30 aves (Gallus gallus) da linhagem Cobb 500, machos, com idade entre 5 e 6 semanas, procedentes de um núcleo criatório localizado no município de 
Uberlândia, MG.

Com a finalidade de marcar os contingentes arteriais, promoveu-se em cada espécime a identificação da artéria isquiática esquerda, localizada na face medial da coxa sob a pele e músculos pubisquiofemoral e flexor crural medial, em intima relação com a face medial do músculo iliofibular. Após dissecação e canulação da artéria, o preenchimento dos vasos foi realizado através da injeção de solução aquosa de Neoprene Látex “450” (Du Pont do Brasil Indústrias Químicas) a 50\%, corada com pigmento específico (Globo Tintas e Pigmentos S.A.).

A fixação dos animais em solução aquosa de formaldeído (LABSYNTH Produtos para Laboratórios Ltda) a 10\%, ocorreu mediante diferentes pontos de aplicações subcutâneas, intramusculares e intracavitárias, bem como, por imersão destes em recipientes contendo a mesma solução por um período mínimo de 48 horas.

Com o intuito de executar a dissecação das artérias mesentéricas cranial e caudal realizou-se abertura da cavidade peritonial em cada um dos espécimes.

A nomenclatura utilizada na presente investigação científica está de acordo com Baumel et $\mathrm{al}^{7}$.

\section{Resultados}

A artéria mesentérica cranial se originou da face lateral direita da aorta descendente, caudalmente à artéria celíaca e após a emissão de seus ramos terminou próximo ao divertículo vitelino em todas as aves.

A artéria ileocecal (Figura 1), identificada nos 30 animais estudadas, foi o primeiro ramo emitido pela artéria mesentérica cranial e, além de distribuir-se pelo íleo e bases e corpos dos cecos direito e esquerdo, enviou ramos ao reto em 18 preparações $(60 \%)$.

A seguir a artéria mesentérica cranial enviou ao longo de sua face lateral direita as artérias jejunais (Figura 1), as quais estiveram presentes em todos os espécimes analisados e variaram de sete a onze artérias, identificando-se sete em 3 casos (10\%), oito em 8 casos $(26,67 \%)$, nove em 10 casos $(33,33 \%)$, dez em 4 casos $(13,33 \%)$ e onze em 5 casos $(16,67 \%)$.

As artérias ileais (Figura 1) surgiram da face lateral esquerda da artéria mesentérica cranial nos 30 animais pesquisados e variaram de duas a quatro artérias, evidenciando-se duas em 18 casos $(60 \%)$, três em 8 casos $(26,67 \%)$ e quatro em 4 casos $(13,33 \%)$.

Verificou-se que uma artéria ileal, mais calibrosa, além de distribuir-se pelo íleo, cedeu ramos aos ápices dos cecos direito e esquerdo em 20 oportunidades $(66,67 \%)$ e somente ao ápice do ceco direito em 8 preparações $(26,67 \%)$.

No atinente à artéria mesentérica caudal, evidenciou-se que esta emergiu da face ventral da aorta descendente e após um curto trajeto bifurcou-se em ramos cranial e caudal em todas as aves analisadas (Figura 2).

O ramo cranial enviou ramos ao reto nos 30 animais estudados (Figura 2), os quais variaram de três a oito ramos, evidenciandose três em 3 casos (10\%), quatro em 8 casos (26,67\%), cinco em 3 casos $(10 \%)$, seis em 11 casos $(36,67 \%)$, sete em 2 casos $(6,67 \%)$ e oito em 3 casos $(6,67 \%)$.

Logo após sua origem, o ramo caudal da artéria mesentérica caudal se bifurcou, distribuindo-se pelas faces laterais direita e esquerda da cloaca em todos os espécimes pesquisados.

Além disso, evidenciou-se que este ramo, antes de sua bifurcação, emitiu um ramo retal em 4 casos (13,33\%), um ramo cloacal em 1 caso $(3,33 \%)$ e um ramo bursocloacal em 1 caso (3,33\%). Já após a bifurcação do ramo caudal da artéria mesentérica caudal, os ramos destinados às faces laterais direita e esquerda da cloaca cederam os ramos retais (Figura 2), cloacais, bursocloacais e bursais, os quais variaram quanto ao número e a presença entre os 
espécimes investigados (Tabela 1). Evidenciaram-se, em todos os espécimes investigados, anastomoses entre ramos da artéria mesentérica cranial e ainda entre ramos desta e da artéria mesentérica caudal, representadas, respectivamente, pelas anastomoses das artérias jejunais e ileais entre si e da artéria ileocecal com o ramo cranial da artéria mesentérica caudal.

No que diz respeito às anastomoses entre ramos das artérias mesentérica cranial e celíaca, observou-se que estas ocorreram entre a primeira artéria jejunal e artéria duodenojejunal em 28 oportunidades (93,33\%), entre a artéria ileocecal e o ramo ileocecal da artéria celíaca em 12 observações $(40 \%)$, entre a artéria ileocecal e o ramo ileocecal da artéria pancreaticoduodenal em 8 preparações $(26,67 \%)$ e entre a artéria ileal e o ramo ileocecal da artéria pancreaticoduodenal nos 30 animais estudados.

\section{Discussão}

Buscando informes referentes às artérias mesentéricas, além da literatura clássica ${ }^{8,9}$, foram compilados relatos a partir da literatura específica, tanto na espécie Gallus gallus ${ }^{10,11,12,13,14,15}$, como em outras espécies de aves $16,17,18,19,20,21,22,23$.

No referente à origem da artéria mesentérica cranial, observou-se nas aves estudadas que esta se originou a partir da aorta descendente, caudalmente à artéria celíaca, concordando com os informes da literatura ${ }^{8,11,17,20,22,23,15}$. Ainda, conforme relataram Gonçalves et al. ${ }^{18}$ e Machado et al. ${ }^{19}$ esta origem se deu pela face lateral direita da aorta descendente.

Após sua origem, a artéria mesentérica cranial emitiu a artéria ileocecal ${ }^{8,10,11,17,19,21,22,9,7}$. A presença de mais de uma artéria ileo-cecal ${ }^{3}$, bem como a possibilidade de ausência desta, mencionada na espécie Meleagris gallopavo ${ }^{17}$ não foram evidenciadas neste estudo.

Corroborando os informes de Schummer ${ }^{9}$, Baumel $^{8}$, Campos et al. ${ }^{10}$, Pinto, Ribeiro e Souza ${ }^{20}$, De Silvio ${ }^{17}$, Gonçalves et al. ${ }^{18}$, Machado et al. ${ }^{19}$, Santana et al. ${ }^{22}$, Santos et al. ${ }^{23}$, Carneiro e Silva et al. ${ }^{11}$ Sampaio et al. ${ }^{21}$ e Severino et al. ${ }^{15}$ a artéria mesentérica cranial enviou um número variável de artérias jejunais. Além disso, conforme descreveram Baumel $^{8}$, Campos et al. ${ }^{10}$, Machado et al. ${ }^{19}$, Santana et al. ${ }^{22}$, Santos et al. ${ }^{23}$, Carneiro e Silva et al. ${ }^{11}$ e Severino et al. ${ }^{15}$, a artéria emitiu as artérias ileais, as quais também variaram quanto ao número. A emissão de números variáveis destas artérias determinou um caráter de individualidade a cada espécime investigado.

A presença de uma calibrosa artéria jejunal como primeiro ramo da artéria mesentérica cranial, mencionada nas espécies Rhea americana ${ }^{18}$ e Phoenicopterus ruber chilensis ${ }^{19}$, respectivamente, e ainda das artérias duodenojejunal e ileocecal distal na espécie Coturnix coturnix japonica ${ }^{21}$ não foi identificada na presente investigação científica. Ainda, não foi verificada a divisão desta artéria em três ramos conforme descrito na espécie Cairina moshata ${ }^{20}$, ressaltando a diversidade de comportamentos que podem ser exibidos pela artéria em diferentes espécies de aves.

A artéria mesentérica cranial terminou atingindo o intestino próximo ao divertículo vitelino ${ }^{8,11,15}$, contrapondo as descrições na espécie Meleagris gallopavo ${ }^{17}$, onde que a artéria terminou no primeiro ramo destinado ao ápice dos cecos e os relatos na espécie Rhea american $^{18}$ nos quais a artéria termina em ramos ileocecais.

Com relação à distribuição da artéria mesentérica cranial, além da irrigação do jejuno ${ }^{8,11,18,20,9,15}$ e do íleo ${ }^{8,11,20,9,15}$, evidenciouse nas aves estudadas que a artéria mesentérica cranial foi responsável pelo suprimento sanguíneo dos $\operatorname{cecos}^{10,11,18,20,15} \mathrm{e}$ do reto ${ }^{20}$. A irrigação do íleo pela artéria mesentérica cranial, relatada por Gonçalves et al. $^{18}$ na espécie Rhea americana, não foi evidenciada neste estudo.

No que tange a origem da artéria mesentérica caudal, conforme relataram Schummer ${ }^{9}$, Baumel ${ }^{16}$, Pinto, Ribeiro e Souza $^{20}$, De Silvio ${ }^{17}$, Santana et al..$^{22}$, Santos et al. ${ }^{23}$, Carneiro e Silva et al. ${ }^{11}$ e Severino et al. ${ }^{15} \mathrm{e}$ da mesma forma nas aves da linhagem 
Cobb 500, esta se originou da aorta descendente, diferindo das observações nas espécies Rhea americana ${ }^{18}$ e Phoenicopterus ruber chilensis $^{19}$, nas quais artéria se originou da artéria sacral.

Coadunando os dados da literatura, após sua origem, a artéria mesentérica caudal se bifurcou em ramos cranial e caudal $^{8,10,11,17,18,19,20,22,23,15}$. O ramo cranial da artéria mesentérica caudal, conforme as informa-ções concedidas por De Silvio ${ }^{17}$, Gonçalves et al. ${ }^{18}$, Machado et al. ${ }^{19}$, Carneiro e Silva et al. ${ }^{11}$ e Severino et al. ${ }^{15}$, enviou ramos ao reto e estes variaram quanto ao número. Os ramos para o íleo e cecos, descritos também no Gallus gallus ${ }^{11,15}$, não foram evidenciados na linhagem estudada, destacando-se a variedade de arranjos que podem ser con-figurados por este ramo.

O ramo caudal da artéria mesentérica caudal se bifurcou logo após sua origem e distribuiu-se pelas faces laterais direita e esquerda da cloaca, fato não notificado na literatura consultada. Este ramo, durante o seu trajeto, enviou os ramos retais e cloacais ${ }^{11,23,15}$, bem como os ramos bursocloacais e bursais ${ }^{12,13,14}$.

No condizente à distribuição da artéria mesentérica caudal, observou-se que a artéria forneceu suprimento sanguíneo ao reto $^{8,16,10,11,17,18,19,22,23,9,15}$, à cloaca ${ }^{10,11,20,23,15} \mathrm{e}$ à bolsa cloacal ${ }^{16,11,12,20,13,14}$. A irrigação do íleo pela artéria mesentérica caudal, descrita por Campos et al. ${ }^{10}$, Carneiro e Silva et al. ${ }^{11} \mathrm{e}$ Severino et al. ${ }^{15}$, bem como sua distribuição pelos cecos, evidenciada por Carneiro e Silva et al. ${ }^{11}$ e Severino et al. ${ }^{15}$ em estudos com outras linhagens de Gallus gallus, não foram verificadas nas aves da linhagem estudada.
Com relação às anastomoses entre ramos da artéria mesentérica cranial, as observações nas aves investigadas concordaram com as alusões dos autores consultados $^{8,11,15}$, sendo evidenciada a anastomose das as artérias jejunais e ileais entre si em todos os espécimes analisados.

As anastomoses entre as artérias mesentéricas cranial e caudal, ocorreram especificamente entre a artéria ileocecal e o ramo cranial da artéria mesentérica cauda ${ }^{8,10.11 .17,15}$. A anastomose do ramo cranial da artéria mesentérica caudal diretamente com a artéria mesentérica cranial, descrita na espécie Meleagris gallopavo ${ }^{17}$, bem como a presença de um longo arco anastomótico comunicando a primeira artéria jejunal ao ramo cranial da artéria mesentérica caudal, relatada na espécie Rhea americana $^{18}$, não foram identificadas na espécie investigada.

Concordando com os relatos dos autores consultados ${ }^{11,15}$, ocorreram anastomoses entre as artérias mesentérica cranial e celíaca, sendo que, como consignado por $\mathrm{Baumel}^{8}$ esta se deu entre a primeira artéria jejunal e ramos da artéria duodenojejunal e entre a artéria ileal e o ramo ileocecal da artéria pancreaticoduodenal. Além disso, a artéria ileocecal se anastomosou com o ramo ileocecal da artéria pancreaticoduodenal ${ }^{21} \mathrm{e}$ ramos ileocecais da artéria celíaca ${ }^{11,15}$. Em contrapartida, não foi verificada nas aves estudadas a anastomose entre a artéria jejunal e ramos da artéria hepática direita ${ }^{8}$.

\section{Conclusões}

Com base na análise dos resultados concluiu-se que em aves (Gallus gallus) da

Tabela 1 - Número de ramos (\%) emitidos após a bifurcação do ramo caudal da artéria mesentérica caudal pelos ramos destinados às faces lateral direita (Rd) e esquerda (Re) da cloaca em aves da linhagem Cobb 500. Uberlândia, 2004

\begin{tabular}{lcccccccc}
\hline \multirow{2}{*}{ Ramos } & \multicolumn{9}{c}{ Rd } & \multicolumn{9}{c}{ Re } \\
\cline { 2 - 8 } \multicolumn{1}{c}{1} & 2 & 3 & Total & 1 & 2 & 3 & Total \\
\hline Retais & 60 & 16,67 & 10 & 86,67 & 50 & 30 & 10 & 90 \\
Cloacais & 36,67 & 3,33 & & 40 & 23,33 & 13,33 & 36,67 \\
Bursocloacais & 10 & & 10 & 6,67 & & 6,67 \\
Cloacais & 3,33 & & & 3,33 & 6,67 & & 6,67 \\
\hline
\end{tabular}




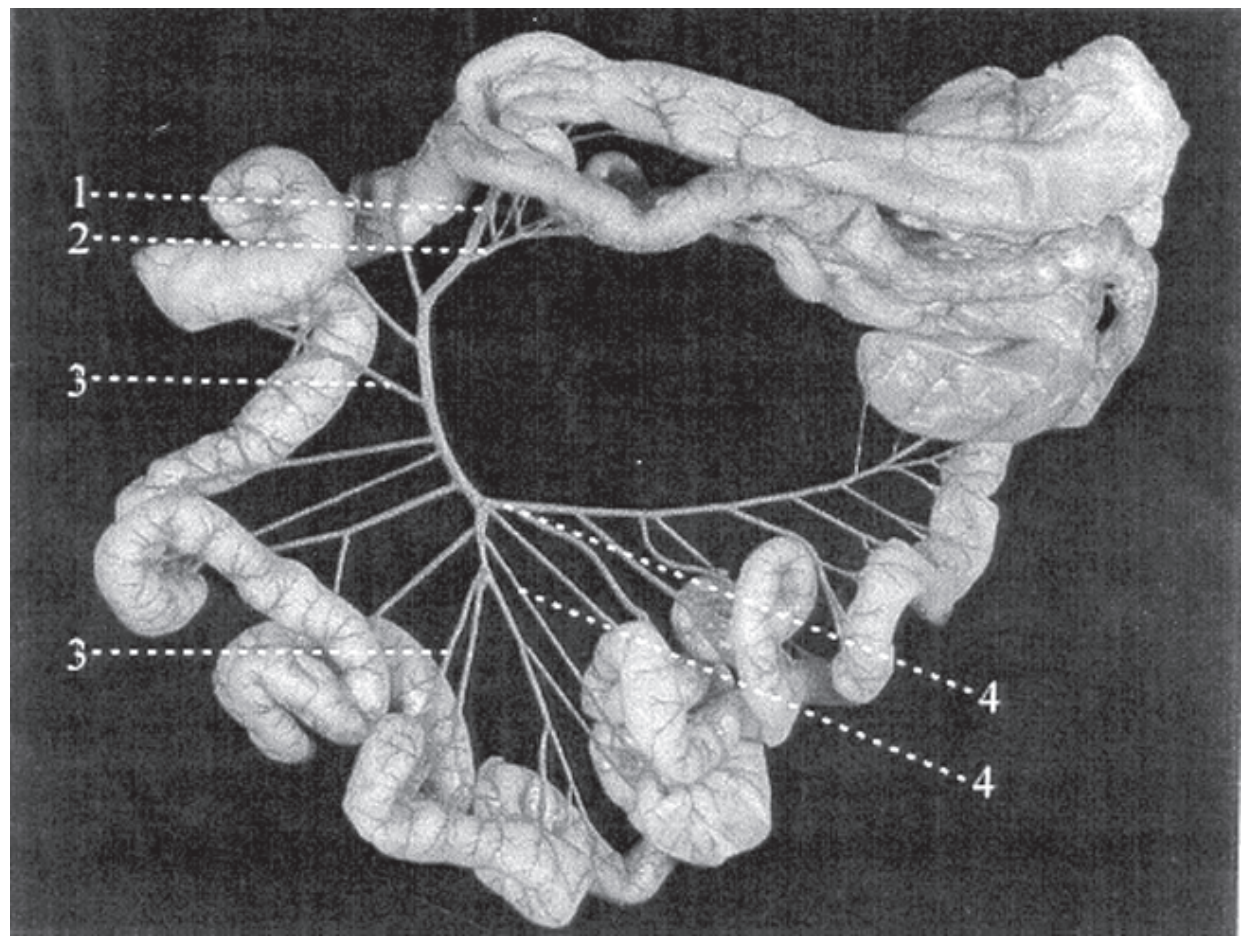

Figura 1 - Fotografia ilustrando a artéria mesentérica cranial (1) emitindo as artérias ileocecal (2), jejunais (3) e ileais (4) em ave (Gallus gallus) da linhagem Cobb 500

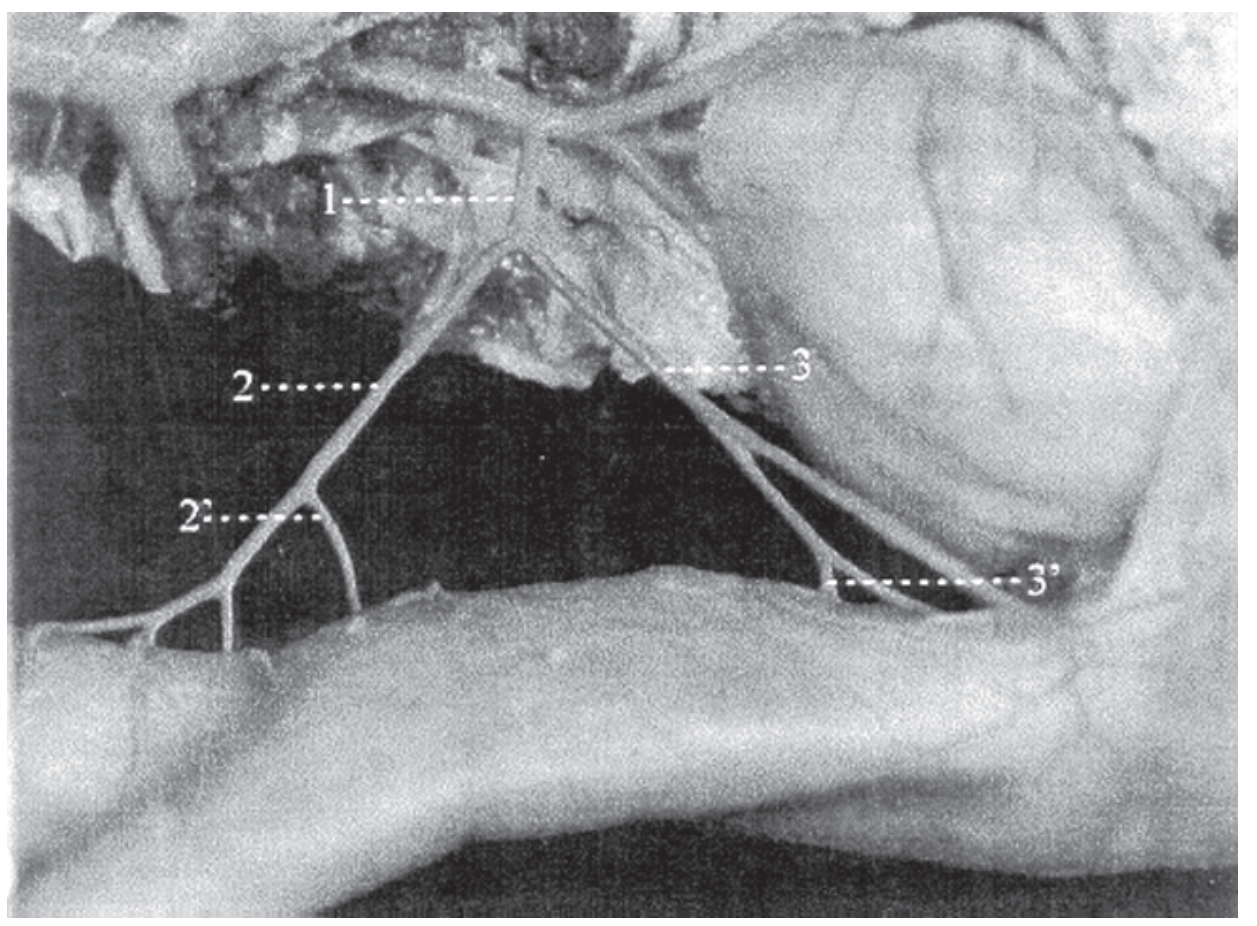

Figura 2 - Fotografia da vista lateral esquerda da cavidade peritonial de ave(Gallus gallus) da linhagem Cobb 500 caracterizando a artéria mesentérica caudal (1) se bifurcando em ramos cranial (2) e caudal (3). Além disso, observa-se o ramo cranial cedendo um ramo ao reto ( $2^{\prime}$ ) e o ramo caudal enviando um ramo retal (3's) 
linhagem Cobb 500:

A artéria mesentérica cranial emergiu da face lateral direita da aorta descendente caudalmente à artéria celíaca e através das artérias ileocecal, jejunais e ileais distribuiu-se pelo jejuno, íleo, cecos e reto, terminando próximo ao divertículo vitelino;

A artéria mesentérica caudal se originou da face ventral da aorta descendente e após curto trajeto bifurcou-se em ramos cranial e caudal, distribuindo-se pelo reto, cloaca e bolsa cloacal;

Ocorreram anastomoses entre ramos da artéria mesentérica cranial e ainda entre ramos desta e das artérias mesentérica caudal e celíaca;

As artérias mesentéricas cranial e caudal apresentaram características distintas das observadas em diferentes linhagens de Gallus gallus e em aves de outras espécies.

\title{
Cranial and caudal mesenteric arteries in fowls (Gallus gallus) of the Cobb 500 lineage
}

\begin{abstract}
Theorigins, ramifications and distributions of the cranial and caudal mesenteric arteries were studied in 30 male fowls (Gallus gallus) from Cobb 500lineage, between 5 and 6 weeks old. The arterial vases of the specimens were filled with colored water-based solutionof Neoprene Latex " 450 " at 50\% concentration afterwards they were fixed in a water-based solution of formaldehyde at $10 \%$ concentration. Thecranial mesenteric artery emerged from the right lateral surface of the descending aorta caudally the coeliac artery and through the ileocecal, jejunal and ileal arteries distributing itself by the jejunum, ileum, cecum and rectum to end next to the vitelline diverticulum. The caudal mesenteric artery originated from the ventral surface of the descending aorta and soon divided into cranial and caudal branches, distributed by the rectum, cloaca and cloacal bursa. There were anastomoses among branches of the cranial mesenteric artery and also among branches of the cranial mesenteric artery and branches of the caudal mesenteric and coeliac arteries.
\end{abstract}

Key-words: Mesenteric

\section{Referências}

1 MACHADO, J. N. Tendências atuais e futuras no uso de colonizadores bacterianos intestinais na avicultura industrial. In: ENCONTRO INTERNACIONAL DE CIÊNCIAS AVIÁRIAS, 4. 2000, Uberlândia. Anais... Uberlândia: Faculdade de Medicina Veterinária da Universidade Federal de Uberlândia, 2000. p. 18-27.

2 LUCHESI, J. B. Efeito da ambiência na performance produtiva de aves. In: SIMPÓSIO DE NUTRIÇÃO E MANEJO DE AVES E SUÍNOS DO TRIÂNGULO, 1. 1998, Uberlândia, Anais... Uberlândia: Universidade Federal de Uberlândia, 1998. p. 35-42.

3 HISTÓRIA DA AVICULTURA. Um pouco da história da avicultura. Disponível em: <http:// planeta.terra.com.br/negocios/avebrusque/AveBrusque/ histotia.htm >. Acesso em: 30 maio 2002.

4 COBB VANTRESS BRASIL. A empresa. Disponível em: < http://www.cobb.com.br/naveg.htm > . Acesso em: 01 maio 2002.
5 KING, A.S. Introdução às aves. In: GETTY, R. Sisson/ Grossman anatomia dos animais domésticos. 5. ed. Rio de Janeiro: Guanabara Koogan, 1986. v. 2, p. 1677 1679.

6 DIDIO, L. J. A. Variação anatômica. In: GETTY, R. Sisson/Grossman anatomia dos animais domésticos. 5. ed. Rio de Janeiro: Guanabara Koogan, 1986. v. 1, p. 14-18.

7 BAUMEL, J. J.; et al. Handbook of avian anatomy: nomina anatomica avium. 2.ed. Cambridge: Nuttall Ornithological Club, 1993. 779p.

8 BAUMEL, J. J. Coração e vasos sanguíneos das aves. In: GETTY, R. Sisson/Grossman anatomia dos animais domésticos. 5. ed. Rio de Janeiro: Guanabara Koogan, 1986. v. 2, p. 1842-1880.

9 SCHUMMER, A. Circulatory system. In: Anatomy of the domestic birds. Berlin: Verlag Paul, 1977. v. 5, p. 85-107.

10 CAMPOS, D. B.; et al. Origins and distribution of cranial and caudal mesenteric arteries in fowl (broiler 
breeders, lineage Ross). In: CONGRESO PANAMERICANO DE CIENCIAS VETERINARIAS, 16. 1998, Santa Cruz de la Sierra. Memorias. Santa Cruz de la Sierra: Asociación Panamericana de Ciencias Veterinarias, 1998. p. 215

11 CARNEIRO E SILVA; F. O.; et al. Origens e distribuições das artérias mesentéricas cranial e caudal em aves (Gallus gallus) da linhagem Avian Farms. Bioscience Journal, v. 17, n. 2, p. 89-99, 2001.

12 PINTEA, V.; CONSTANTINESCU, Gh.M.; RADU, C. Vascular and nervous supply of bursa of Fabricius in the hen. Acta Veterinaria Academiae Scientiarum Hungaricae, v. 17, n. 3, p. 263-268, 1967.

13 SANTANA, M. I. S.; CARNEIRO E SILVA, F. O.; SEVERINO, R. S.; SANTOS, A. L. Q.; DRUMMOND, S. S.; BOMBONATO, P. P. Irrigação da bolsa cloacal, em aves reprodutoras, da linhagem Peterson (Gallus gallus domesticus). Brazilian Journal of Veterinary Research and Animal Science, v. 36, n. 2, 1999. Disponível em: <http://www.scielo.br/ scielo.php?script $=$ sci serial\&pid $=1413$ 9596\&lng $=$ pt\&nrm $=$ iso $>$. Acesso em: 17 set. 2001.

14 SANTANA, M. I. S.; CARNEIRO E SILVA, F. O.; SEVERINO, R. S.; SANTOS, A. L. Q.; DRUMMOND, S. S.; BOMBONATO, P. P. Vascularização arterial da bolsa cloacal em Gallus gallus domesticus (matrizes de corte Avian Farms). Brazilian Journal of Veterinary Research and Animal Science, v. 37, n. 2, 2000 a. Disponível em: <http://www.scielo.br/ scielo.php?script $=$ sci serial \&pid $=1413$ 9596\&lng $=$ pt\&nrm $=$ isso $>$. Acesso em: 17 set. 2001.

15 SEVERINO, R. S.; et al. Origem e distribuição das artérias mesentéricas cranial e caudal em aves (Gallus gallus) da linhagem Label Rouge. Arquivo de Ciências Veterinárias e Zoologia da Universidade Paranaense, v. 4, n. 2, p. 163-168, 2001.

16 BAUMEL, J. J. Functional morphology of the tail of the pigeon Columba livia. Advances in Anatomy, Embriology, and Cell Biology, v. 110, p. 63-68, 1988.

17 DE SILVIO, M. M. Vascularização arterial do proventrículo gástrico, ventrículo gástrico e alças intestinais do peru (Meleagris gallopavo, Linnaeus, 1758). 1999. 79 f. Dissertação (Mestrado) - Faculdade de Medicina Veterinária e Zootecnia, Universidade de São Paulo, São Paulo, 1999.

18 GONÇALVES, P. R.; et al Comportamento anatômico das artérias mesentéricas na ema (Rhea americana). Brazilian Journal of Morphological Sciences, v. 17, p. 226-227, 2000. Suplemento.

19 MACHADO, G. V.; et al Aspectos anatômicos das artérias mesentéricas em flamingos (Phoenicopterus ruber chilensis). Brazilian Journal of Morphological Sciences, v. 17, p. 226, 2000. Suplemento.

20 PINTO, M. R. A.; RIBEIRO, A. A. C. M.; SOUZA, W. M. Os arranjos configurados pelas artérias mesentéricas cranial e caudal no pato doméstico (Cairina moshata). Brazilian Journal of Veterinary Research and Animal Science, v. 35, n. 3, p. 107-109, 1998.

21 SAMPAIO, M. A. P.; et al. Distribuição da artéria mesentérica cranial em codorna doméstica (Coturnix coturnix japonica). In: CONGRESSO BRASILEIRO DE MEDICINA VETERINÁRIA, 28. 2001, Salvador. Livro de resumos. Salvador: Sociedade Brasileira de Medicina Veterinária, 2001. p. 48-49.

22 SANTANA, M. I. S.; et al. Origem e distribuição dos ramos das artérias mesentéricas cranial e caudal em galinhas da angola. Brazilian Journal of Morphological Sciences, v. 17, p. 208, 2000 b. Suplemento.

23 SANTOS, A. L. Q.; et al Comportamento das artérias mesentéricas cranial e caudal em pombos (Columba livia gmelin). Brazilian Journal of Morphological Sciences, v. 17, p. 235, 2000. Suplemento. 